\title{
The Effect of Soy Nuts on Glycemic Control, Lipid Profile and Insulin-Resistance in Type 2 Diabetic Patients
}

\author{
Alireza Sedaghat ${ }^{1}$, Hajieh Shahbazian ${ }^{*}$, Fatemeh Haidari², Seyed Peyman Payami ${ }^{1}$, \\ Alireza Jahanshahi ${ }^{1}$, Seyed Mahmoud Latifi ${ }^{1}$ \\ ${ }^{1}$ Health Research Institute, Diabetes Research Center, Ahvaz Jundishapur University of Medical Sciences, Ahvaz, \\ Iran \\ ${ }^{2}$ Nutrition and Metabolic Disease Research Center, Ahvaz Jundishapur University of Medical Sciences, Ahvaz, \\ Iran \\ Email: Alireza.sedaghat51@gmail.com, ${ }^{*}$ hjb shahbazian@yahoo.com, Haidari58@gmail.com, \\ peyman_payami1@yahoo.com, dr.a.jahanshahi@yahoo.com, sml1381@yahoo.com
}

Received 6 January 2015; accepted 16 January 2015; published 21 January 2015

Copyright (C) 2015 by authors and Scientific Research Publishing Inc.

This work is licensed under the Creative Commons Attribution International License (CC BY).

http://creativecommons.org/licenses/by/4.0/

c) (i) Open Access

\section{Abstract}

Background: Type 2 diabetes has a high prevalence and a growing trend. The use of a proper diet treatment is one of the therapeutic approaches of patients. The use of the soy has shown the effective results in glycemic control of patients with type 2 diabetes; however, data are paradoxical. The present study aimed to examine the effectiveness of soy nuts on glycemic control, blood pressure and lipid profile and insulin-resistance of the diabetic patients. Methods: In this case-control study 69 type 2 diabetic patients were randomly divided into two groups: intervention $(n=35)$ and control $(n=34)$. The patients in the intervention group substituted 60 grams of soy nuts as a part of the daily protein requirement for eight weeks. In contrast, the patients in the control group received usual diet of diabetes (no soy). The drugs received by patients had not been changed during the intervention period. Before and at the end of the intervention, Hemoglobin A1c (HbA1c), fasting plasma glucose (FPG), serum insulin levels, insulin-resistance, systolic and diastolic blood pressure, low density lipoprotein-cholesterol (LDL-c), high density lipoprotein-cholesterol (HDL-c) and total cholesterol, and triglycerides (TG) were measured in the patients. Insulin-resistance was calculated by Homeostatic model assessment-IR formula (HOMA-IR). Results: Soy consumption significantly lowered FPG $(P=0.03)$, HbA1c $(P<0.01)$, plasma insulin levels $(P=$ 0.01), insulin-resistance $(P=0.01)$, total cholesterol $(P<0.01)$ and LDL-c $(P=0.01)$, but did not have any significant effect on systolic blood pressure $(P=0.4)$, diastolic blood pressure $(P=0.2)$, HDL-c $(P=0.4)$ and TG $(P=0.2)$. Conclusion: Consumption of soy nuts in type 2 diabetic patients

*Corresponding author.

How to cite this paper: Sedaghat, A., Shahbazian, H., Haidari, F., Payami, S.P., Jahanshahi, A. and Latifi, S.M. (2015) The Effect of Soy Nuts on Glycemic Control, Lipid Profile and Insulin-Resistance in Type 2 Diabetic Patients. Open Journal of Endocrine and Metabolic Diseases, 5, 1-7. http://dx.doi.org/10.4236/ojemd.2015.51001 
can cause an improvement in the glycemic control and insulin-resistance, and the lipid profile does not have any significant effect on blood pressure.

\section{Keywords}

Type 2 Diabetes, Soy Nuts, Blood Glucose, Blood Pressure, Lipid Profile

\section{Introduction}

Diabetes mellitus (DM) involves a group of metabolic disorders with various etiologies which leads to increased blood sugar and impaired glucose, fat and protein metabolism caused by the decreased secretion or activity of insulin or both [1]. According to the American Diabetes Association in 2012, the diabetes' costs have been estimated over 245 billion dollars a year [2].

Modification of the lifestyle such as physical activity and a proper food diet is early action in controlling and preventing the type 2 diabetes disease [3].

The use of the cheap and available food ingredients, lowering blood glucose and improving metabolic condition, is an ideal and optimal action. Impressive results have been recorded for consuming the soy nuts and soy products [4] [5]. Soybeans contain complex carbohydrates, protein, fiber, oligosaccharides, phytosterols, saponins, lectins, isoflavones, phytic acid, trypsin inhibitor, and minerals [4] [5].

The content of complex carbohydrates and dietary fibers in soy generates its low glycemic index, which can be useful in diabetic patients.

Various studies have reported effective results in improving blood sugar disorders, lipid profile and blood pressure due to use of different combinations of soy [4] [5]. And many studies have found that soy can reduce glycemia after meals, hyper triglyceridemia, total cholesterol, LDL-c, oxidative stress and other risk factors for cardiovascular disease [4] [6] [7]; however, the findings are controversial [8].

Plant sources are rich in natural antioxidants and used for control and treatment of many diseases. In animal models and clinical research the glucose-lowering effect in many of plant sources, such as soy, has been assessed and confirmed.

The present study aimed to evaluate the effectiveness of edible soy nuts on the glycemic control, lipid profile, insulin-resistance and blood pressure in type 2 diabetic patients.

\section{Methods}

\subsection{Study Design}

The randomized controlled trial (RCT) was conducted on the patients with type 2 diabetes mellitus referring to the Golestan hospital diabetes clinic in Ahvaz City during 2013-2014.

The inclusion criteria were Hemoglobin A1c (HbA1c) < 8.5, Fasting Plasma Glucose (FPG) < 250, and Body Mass Index $(\mathrm{BMI})<35$ and the exclusion criteria were patients treated with insulin, patients with cardiac, renal or hepatic failure, and the history of allergy to soy products. A simple sequential sampling method was performed. The two groups were matched in terms of age, sex, and BMI, and were divided randomly into two groups: the intervention group $(n=35$ people) and the control group $(n=34$ people). In intervention group some of daily protein requirements were substituted by soy nut.

In the beginning of the study, all patients were undergoing dietary consultations for Therapeutic Lifestyle Changes (TLC) and diabetes dietary recommendations. Furthermore, the type and dosage of medication of the patients as well as their physical activity were not changed during the study. At the beginning and the end of the study, the dietary intakes by patients were evaluated using the 24-hour dietary recall questionnaire for three days.

The soy nuts in 30 gram packs were given to people in the intervention group and they were asked to consume 60 grams of soy nuts daily in morning and afternoon snacks for eight weeks. At the beginning and the end of the study the basic information about the patients, including age, sex, weight, BMI, waist circumference (in minimum diameter), and hip circumference (in maximum diameter) were measured. 


\subsection{Biochemical Assessments}

At the beginning and the end of the study 5 cc of the cubital venous blood samples were taken from all patients after 10 hours of fasting to study the biochemical indices including: HbA1c; FPG, serum insulin level, High density lipoprotein-cholesterol (HDL-c) and Total Cholesterol and triglyceride (TG) of patients and immediately moved to the lab. FPG, TG, total cholesterol and HDL-c were measured by enzymatic method using standard kits (Pars Azma Co., Iran) auto-analyzer (BT 3000, France) and HbA1c were measured by the capillary hemoglobin electrophoresis method, and plasma insulin levels were measured by Elisa method. Low density lipoprotein-cholesterol (LDL-c) was calculated using Fridewald's formula:

$$
\text { LDL-c }=\text { total cholesterol }-(\text { TG/5 + HDL-c })
$$

Insulin-resistance was measured by Homeostasis model assessment (HOMA-IR) index, which includes the following formula:

$$
\text { [HOMA-IR :] }[(((\mathrm{FPG}) \mathrm{mmol} / \mathrm{l}) \times(\text { fasting serum insulin }) \mu \mathrm{IU} / \mathrm{ml}) / 22.5]
$$

Consecutively, with a time distance of half an hour two times, the blood pressure levels from left arms of patients (in a sitting position) were measured and their average was recorded. Patients were not anxious and have not utilized alcohol, tea, and coffee and sports activity at three hours before measurement of blood pressure. Moreover, the fabric meter with an accuracy of $1 \mathrm{~mm}$ and an analog scale with the accuracy of $0.1 \mathrm{~kg}$ were used for metric measuring, and to determine the weight, respectively.

\subsection{Statistical Analysis}

The data were collected in a checklist and analyzed using the SPSS version 17. Data were expressed in the form of mean and standard deviation (SD). To check the normal distribution of the data the Kolmogorov-Smirnov test was used.

Mean changes between the case and control groups were statistically determined by independent t-test for intergroup comparison and by paired t-test for intragroup comparison. The significance level was considered equal to $P<0.05$.

\section{Results}

Basic and demographic information about patients have been presented in Table 1. The results of this table shows that patients were been matched well based on the desired variables in two groups $(P>0.05)$.

Table 2 shows the amount of calories and food molecule compounds consumed during the study. In general after intervention no significant difference was seen between the two groups in terms of the amount of calorie intake $(P=0.2)$. Compared to the control group, the intervention group indicated significantly larger amount of carbohydrates $(P>0.05)$ and fiber $(P=0.03)$ and less amount of protein and fat $(P>0.05)$.

Before starting the intervention, patients had a uniform distribution in terms of ten desired variables in both group and no significant difference has been observed between them excepting the diastolic blood pressure $(P=$ 0.02) with optimal distribution.

Table 1. Basal demographic information of patients.

\begin{tabular}{cccc}
\hline Variable & Intervention group $(\mathbf{n}=\mathbf{3 5})$ & Control group $(\mathbf{n}=\mathbf{3 4})$ & $\boldsymbol{P}$ \\
\hline Gender (person) & & & 0.6 \\
Male & 16 & 15 & \\
Female & 19 & 19 & \\
Mean age (year) $\pm \mathrm{SD}$ & $49.9(9.2)$ & $50.2(8.3)$ & 0.3 \\
Mean waist circumference $(\mathrm{cm}) \pm \mathrm{SD}$ & $101(7.1)$ & $100.3(9.8)$ & 0.3 \\
Mean hip circumference $(\mathrm{cm}) \pm \mathrm{SD}$ & $105.4(7.6)$ & $103.1(9)$ & 0.4 \\
Mean weight $(\mathrm{kg}) \pm \mathrm{SD}$ & $74.4(15.5)$ & $76.3(10.1)$ & 0.2 \\
Mean BMI $\left(\mathrm{kg} / \mathrm{m}^{2}\right) \pm \mathrm{SD}$ & $29(3.2)$ & $28.2(3.3)$ & 0.6 \\
\hline
\end{tabular}


Table 2. Food ingredients and caloric intake in the two groups after two months of intervention.

\begin{tabular}{ccccc}
\hline \multirow{2}{*}{ Variable } & \multicolumn{2}{c}{ Mean (SD) } & Mean changes & P \\
\cline { 2 - 4 } & Intervention group (n= 35) & Control group (n = 34) & & \\
\hline Daily calories (kcal) & $1864(457.86)$ & $1737.5(463.3)$ & $126.5+$ & 0.2 \\
Carbohydrates (gr) & $383.5(168.5)$ & $303.7(145.4)$ & $79.8+$ & 0.07 \\
Fat (gr) & $31.9(28.7)$ & $40.8(21.7)$ & $8.9-$ & 0.06 \\
Protein (gr) & $39.8(39.1)$ & $53.3(30.1)$ & $13.5-$ & 0.1 \\
Fiber (gr) & $43.1(34.3)$ & $23.4(27.8)$ & $19.7+$ & $\underline{0.03}$ \\
\hline
\end{tabular}

At the end of therapeutic intervention (after eight weeks) the paired t-test did not show any significant change in the variables among the patients in the control group (Table 3).

In the intervention group, the consumption of Soy nuts created a significant effect on studied variables (except HDL-c and TG levels) so that the changes in FPG, HbAlc, the plasma insulin level, cholesterol and LDL-c levels, and the insulin-resistance were statistically significant (Table 3).

After the intervention, an intergroup comparison by independent t-test showed significantly positive effect of soya on the five parameters: FPG, HbA1c, insulin-resistance, total cholesterol and low density cholesterol, resulting to reduce amount of above mentioned variables. Furthermore, serum insulin level in the intervention group was less than the control group, but the changes were not significant (Table 3).

\section{Discussion}

Presenting results suggest that consumption of soy nuts lowered FPG $(P=0.03)$, HbA1c $(P=0.01)$, insulin-resistance $(P=0.01)$, total cholesterol $(P=0.01)$, and LDL-c $(P=0.01)$, however it did not have a significant effect on systolic blood pressure $(P=0.4)$, diastolic blood pressure $(P=0.2)$, HDL-c $(P=0.4)$, TG $(P=0.2)$ and plasma insulin levels $(P=0.01)$.

Hermansen et al. showed that the soy consumption could reduce LDL-c, TG, and total cholesterol by $10 \%$, $22 \%$, $8 \%$, respectively when type 2 diabetic patients consumed daily 70 grams soys for a period of six weeks. They report further no positive effect of this especial diet on HbA1c and blood pressure of patients [4], what could erroneously happen due short time of treatment necessary for HbA1c changing.

Furthermore, Jayagopal et al. reported decreases of $8.1 \%, 0.6 \%, 4.1 \%$ and $7.1 \%$ in the insulin level, HbA1c, total cholesterol, and LDL-c, respectively in 32 diabetic postmenopausal women consuming 30 grams of soy phytoestrogens daily for 12 weeks. They also reported no effect of soy intake on blood pressure TG and HDL-c [9], which is similar to our data.

Chang et al. showed further that daily consumption of 69 grams soy by the type II diabetic for four weeks could decrease triglycerides and FPG, and postprandial plasma glucose, as well [6]; which is consistent with our finding [10]. Liao et al. showed that 45 grams of soy added to low-calorie diet (1200 kcal) for eight weeks could lowers LDL-c and total cholesterol, which has no effect on the recovery of TG [10]. Indeed, we experienced in our suggestive diet in the present report similar results.

Shahbazian et al. showed beneficial effects of daily 25 grams of soy protein isoflavones for three months on the level of lipid and concentration of blood glucose in type 2 diabetic patients. They results showed that FPG, HbA1c, total cholesterol and TG were considerably improved, but significant changes were not seen in the levels of HDL-c, weight and blood pressure [5].

In another study, Kwak et al. showed that soy consumption for 12 weeks improved fasting and 2-hour blood glucose in type 2 diabetic patients compared to control group, but did not have any significant effect on reducing the level of HbA1c [11]. A Meta-analysis performed by Reynolds et al. confirmed, the positive effect of the soy consumption on total cholesterol, LDL-c, TG and HDL-c [12] that is in concordance with our finding.

Our results showed that taking soy nuts could not improve blood pressure. However, in some studies soy consumption has had a lowering effect on blood pressure. Furthermore, Welty et al. reported in a study of 56 menopausal women with either hypertension or normal blood pressure, that consumption of $56 \mathrm{~g}$ of soy nuts for eight weeks reduced systolic blood pressure and diastolic blood pressure by $9.9 \%$ and $6.8 \%$, respectively in the hypertensive patients and $5.2 \%$ and $2.7 \%$ in individuals with normal blood pressure [13]. Nasca et al. also 
Table 3. The comparison between the studied variables before and after intervention in the case and control groups.

\begin{tabular}{|c|c|c|c|c|c|c|c|c|c|}
\hline \multirow{3}{*}{ Variable } & \multicolumn{8}{|c|}{ Mean (SD) } & \multirow{3}{*}{$P^{1}$} \\
\hline & \multicolumn{4}{|c|}{ Intervention group } & \multicolumn{4}{|c|}{ Control group } & \\
\hline & Before & After & $\boldsymbol{P}$ & Dif & Before & After & $\boldsymbol{P}$ & Dif & \\
\hline Fasting blood glucose (mg/dl) & $165.5(51)$ & $148.2(49)$ & $<0.01$ & -17.3 & $161.7(41.2)$ & $167.5(42.3)$ & 0.3 & +6.1 & 0.03 \\
\hline HbA1c (mg/dl) & $7.7(0.7)$ & $7.2(0.7)$ & $<0.01$ & -0.5 & $7.7(0.5)$ & $7.8(0.6)$ & 0.09 & +0.1 & $<0.01$ \\
\hline Insulin levels (mg/dl) & $9.9(3)$ & $9(2.7)$ & $<0.01$ & -0.9 & $10.5(3.4)$ & $10.2(3.1)$ & 0.2 & -0.3 & 0.08 \\
\hline Insulin-resistance & $4.1(1.8)$ & $3.6(1.5)$ & $<0.01$ & -0.5 & $4.9(2.1)$ & $5.1(2)$ & 0.2 & +0.1 & 0.01 \\
\hline Systolic blood pressure (mmHg) & $132.3(16.2)$ & $125.5(14.2)$ & $<0.01$ & -6.8 & $129.8(15)$ & $128.7(13)$ & 0.3 & -1.1 & 0.4 \\
\hline Diastolic blood pressure (mmHg) & $86(7.7)$ & $79(14.1)$ & $<0.01$ & -7.0 & $81.8(6.5)$ & $83.1(6.8)$ & 0.07 & +1.3 & 0.2 \\
\hline Total cholesterol (mg/dl) & $178(30.3)$ & $159.4(29)$ & $<0.01$ & -18.6 & $179.3(25.8)$ & $180.7(24.8)$ & 0.08 & +4.8 & $<0.01$ \\
\hline HDL-cholesterol (mg/dl) & $41.6(6.6)$ & $40.3(6.3)$ & 0.1 & -1.3 & $41.1(7.2)$ & $41.3(6.9)$ & 0.5 & +0.2 & 0.4 \\
\hline LDL-cholesterol & $104.7(22)$ & $90.8(21.7)$ & $<0.01$ & -13.9 & $102.8(22.9)$ & $103.9(20.2)$ & 0.6 & +1.1 & 0.01 \\
\hline TG (mg/dl) & $173.1(63.1)$ & $163.2(49.9)$ & 0.2 & -9.9 & $161.6(52.7)$ & $164.1(48.8)$ & 0.8 & +2.5 & 0.9 \\
\hline
\end{tabular}

FPG: 70 - $130 \mathrm{mg} / \mathrm{dl}$; HbA1c < 7\%; LDL-c < $100 \mathrm{mg} / \mathrm{dl}$; HDL-c $>40 \mathrm{mg} / \mathrm{dl}$ for male, $>50 \mathrm{mg} / \mathrm{dl}$ for female; TG < $150 \mathrm{mg} / \mathrm{dl}$; Cholesterol < 200 $\mathrm{mg} / \mathrm{dl}$; Dif = mean difference between before and after intervention in the case and control groups; $P=$ significance changes within groups; ${ }^{1} P=$ significant changes between groups.

showed that consuming 25 grams of soy for eight weeks in hypertensive postmenopausal women is followed by an average decrease of $9.9 \%$ and $6.6 \%$ respectively in systolic and diastolic blood pressure [14].

It seems that the metabolic disorder of the sugar and insulin-resistance play a pivotal role in the incidence of dyslipidemia and the hypertension in people with type 2 diabetes [15] [16]. The positive effect of the herbal compounds on improving the performance of the pancreatic b-cells has been reported previously [17]. In a study of soy consumption in diabetic mice, Ascencio et al. recorded a reduction in glucagon, insulin and blood sugar [18]. Also Noriega-López et al.'s study showed that consumption of soy protein reduced irritability of pancreatic islets of Langer-Hans as well as hyperinsulinemia [19].

To date, several mechanisms have been described in the effectiveness of soy consumption on controlling blood pressure and lipid and glycemic conditions. Soy has many different compounds; however, contains large amounts of isoflavones, a group of the phytoestrogens, which has to be proved by the rate of the effectiveness of each of these components on lipid and plasma glucose; however generally it seems with pivot effect of estrogen on controlling glycemic conditions the soy causes weight loss, TG, LDL-c and Total cholesterol. This substance also facilitates the removal of glucose by cells and reduces fat tissue [5] [9] [20]. Polysaccharide is another important component of soy. In a small and short- term crossover study conducted on 19 healthy students Tsai et al. showed that consumption of soy polysaccharide did not have an effect on blood lipid profile although improves the glucose tolerance [21]. By improving the molecule level connecting the vascular cell type and consequently improving the performance of vascular endothelial cells and modulating the atherosclerosis and inflammatory process the soy is effective in reducing blood pressure [15]. Soy consumption caused a reduction in some of the chemical intermediaries such as the IL-18, CRP and increasing NO in postmenopausal women with the metabolic syndrome, which was effective in improving performance that could be another mechanism on the effectiveness of soy on blood pressure and vascular health [22].

In addition to soy compound it seems hypoglycemic hypolipidemia effects of soy are dependent on hormonal changes. The lab test showed that flanovid of soy reduces insulin levels, glucagon and leptin in circulation and the effectiveness of soy is associated with BMI so that flanovid did not have any effect on the level of blood triglycerides in normal mice compared to the obese mice [23]. The type of soy consumed also is effective in the success of the soy. In a review study, intake of soy nuts or soy protein improved the cardiac metabolic effects, although a soy nut was superior on soy protein in the results by the researchers and there is a need for more studies in this field [24].

In our study, while patients received the TLC diet instruction the greater percentage of their food calories was carbohydrate and in a lesser extent fat and protein; however they did not have any difference in the amount of 
calories, fat and protein intake at the end of the study. Also in the soy group the average daily fiber was more than the recommended rate (average 48 grams versus the 15 - 20 grams) and more than the control group (14 grams) $(P=0.00)$. It was well illustrated that fiber-filled diet is associated with reducing or preventing many chronic diseases, including cardiovascular disease, increasing blood pressure, enhancement of blood lipid, obesity, diabetes, some cancers, and diseases of the digestive system and enhances immune system [25] [26]. In this study it seems the soy fiber, in addition to its other compounds, have also been able to cause improvement of metabolic disorder in treated patients.

The limitations in our study included the lack of long-term assessments and lack of evaluation of the different dosages of the soya. These issues should be examined in future studies.

\section{Conclusion}

This study showed that an eight-week daily consumption of 60 grams soy nuts caused a significant decrease in the levels of HbA1c, FPG, LDL-c, total cholesterol and insulin-resistance, but it did not have a significant effect on the levels of HDL-c, TG and blood pressure.

\section{Competing Interests}

The authors declare that they have no competing interests.

\section{Authors' Contributions}

HSh, FH designed the study. SPP and AJ supervised and referred patients. AS has done research, data collection and drafted manuscript. SML has done the statistical analysis. All authors edited read and approved the final manuscript. Thanks to all authors for their support and help in this study.

\section{Acknowledgements}

This paper is issued from the thesis of Dr. Alireza Sedaghat. Financial support was provided by Health Research Institute, Diabetes Research Center, Ahvaz Jundishapur University of Medical Sciences. The authors would like to thank all staffs of diabetes research center, Miss Dehghan and Hardani for their help in this study.

\section{References}

[1] Melmed, S., Polonsky, K.S., Reed Larsen, P. and Kronenberg, H.M. (2011) Williams Textbook of Endocrinology: Expert Consult. Elsevier Health Sciences, 1701-1703.

[2] Yang, W., Dall, T.M., Halder, P., Gallo, P., Kowal, S.L. and Hogan, P.F. (2013) Economic Costs of Diabetes in the U.S. in 2012. Diabetes Care, 36, 1033-1046. http://dx.doi.org/10.2337/dc12-2625

[3] Stone, N.J. and Van Horn, L. (2002) Therapeutic Lifestyle Change and Adult Treatment Panel III: Evidence Then and Now. Current Atherosclerosis Reports, 4, 433-443. http://dx.doi.org/10.1007/s11883-002-0047-x

[4] Hermansen, K., Søndergaard, M., Høie, L., Carstensen, M. and Brock, B. (2001) Beneficial Effects of a Soy-Based Dietary Supplement on Lipid Levels and Cardiovascular Risk Markers in Type 2 Diabetic Subjects. Diabetes Care, 24, 228-233. http://dx.doi.org/10.2337/diacare.24.2.228

[5] Shahbazian, H., Reza, A., Javad, S., Heshmatollah, S., Mahmood, L., Ali, A. and Hosain, H.M. (2007) Beneficial Effects of Soy Protein Isoflavones on Lipid and Blood Glucose Concentrations in Type 2 Diabetic Subjects. Saudi Medical Journal, 28, 652-654.

[6] Chang, J.H., Kim, M.S., Kim, T.W. and Lee, S.S. (2008) Effects of Soybean Supplementation on Blood Glucose, Plasma Lipid Levels, and Erythrocyte Antioxidant Enzyme Activity in Type 2 Diabetes Mellitus Patients. Nutrition Research and Practice, 2, 152-157. http://dx.doi.org/10.4162/nrp.2008.2.3.152

[7] Clerici, C., Nardi, E., Battezzati, P.M., Asciutti, S., Castellani, D., Corazzi, N., Giuliano, V., et al. (2011) Novel Soy germ Pasta Improves Endothelial Function, Blood Pressure, and Oxidative Stress in Patients with Type 2 Diabetes. Diabetes Care, 34, 1946-1948. http://dx.doi.org/10.2337/dc11-0495

[8] Liu, Z., Chen, Y., Ho, S.C., Ho, Y.P. and Woo, J. (2010) Effects of Soy Protein and Isoflavones on Glycemic Control and Insulin Sensitivity: A 6-mo Double-Blind, Randomized, Placebo-Controlled Trial in Postmenopausal Chinese Women with Prediabetes or Untreated Early Diabetes. The American Journal of Clinical Nutrition, 91, 1394-1401. http://dx.doi.org/10.3945/ajcn.2009.28813 
[9] Jayagopal, V., Albertazzi, P., Kilpatrick, E.S., Howarth, E.M., Jennings, P.E., Hepburn, D.A., et al. (2002) Beneficial Effects of Soy Phytoestrogen Intake in Postmenopausal Women with Type 2 Diabetes. Diabetes Care, 25, 1709-1714. http://dx.doi.org/10.2337/diacare.25.10.1709

[10] Liao, F.H., Shieh, M.J., Yang, S.C., Lin, S.H. and Chien, Y.W. (2007) Effectiveness of a Soy-Based Compared with a Traditional Low-Calorie Diet on Weight Loss and Lipid Levels in Overweight Adults. Nutrition, 23, 551-556. http://dx.doi.org/10.1016/j.nut.2007.05.003

[11] Kwak, J.H., Kim, M., Lee, E., Lee, S.H., Ahn, C.W. and Lee, J.H. (2013) Effects of Black Soy Peptide Supplementation on Blood Pressure and Oxidative Stress: A Randomized Controlled Trial. Hypertension Research, 36, 1060-1066. http://dx.doi.org/10.1038/hr.2013.79

[12] Reynolds, K., Chin, A., Lees, K.A., Nguyen, A., Bujnowski, D. and He, J. (2006) A Meta-Analysis of the Effect of Soy Protein Supplementation on Serum Lipids. American Journal of Cardiology, 98, 633-640. http://dx.doi.org/10.1016/j.amjcard.2006.03.042

[13] Welty, F.K., Lee, K.S., Lew, N.S. and Zhou, J.R. (2007) Effect of Soy Nuts on Blood Pressure and Lipid Levels in Hypertensive, Prehypertensive, and Normotensive Postmenopausal Women. Archives of Internal Medicine, 167, 10601067. http://dx.doi.org/10.1001/archinte.167.10.1060

[14] Nasca, M.M., Zhou, J.R. and Welty, F.K. (2008) Effect of Soy Nuts on Adhesion Molecules and Markers of Inflammation in Hypertensive and Normotensive Postmenopausal Women. American Journal of Cardiology, 102, 84-86. http://dx.doi.org/10.1016/j.amjcard.2008.02.100

[15] Stas, S.N., El-Atat, F.A. and Sowers, J.R. (2004) Pathogenesis of Hypertension in Diabetes. Reviews in Endocrine and Metabolic Disorders, 5, 221-225. http://dx.doi.org/10.1023/B:REMD.0000032410.75638.da

[16] Mooradian, A.D. (2009) Dyslipidemia in Type 2 Diabetes Mellitus. Nature Clinical Practice Endocrinology \& Metabolism, 5, 150-159. http://dx.doi.org/10.1038/ncpendmet1066

[17] Tabatabaei-Malazy, O., Larijani, B. and Abdollahi, M. (2012) A Systematic Review of in Vitro Studies Conducted on Effect of Herbal Products on Secretion of Insulin from Langerhans Islets. Journal of Pharmaceutical Sciences, 15, 447-466.

[18] Ascencio, C., Torres, N., Isoard-Acosta, F., Gómez-Pérez, F.J., Hernández-Pando, R. and Tovar, A.R. (2004) Soy Protein Affects Serum Insulin and Hepatic SREBP- ${ }_{-1}$ mRNA and Reduces Fatty Liver in Rats. Journal of Nutrition, 134, 522-529.

[19] Noriega-López, L., Tovar, A.R., Gonzalez-Granillo, M., Hernández-Pando, R., Escalante, B., Santillán-Doherty, P., et al. (2007) Pancreatic Insulin Secretion in Rats Fed a Soy Protein High Fat Diet Depends on the Interaction between the Amino Acid Pattern and Isoflavones. Journal of Biological Chemistry, 282, 20657-20666. http://dx.doi.org/10.1074/jbc.M701045200

[20] Cederroth, C.R. and Nef, S. (2009) Soy, Phytoestrogens and Metabolism: A Review. Molecular and Cellular Endocrinology, 304, 30-42.

[21] Tsai, A.C., Mott, E.L., Owen, G.M., Bennick, M.R., Lo, G.S. and Steinke, F.H. (1983) Effects of Soy Polysaccharide on Gastrointestinal Functions, Nutrient Balance, Steroid Excretions, Glucose Tolerance, Serum Lipids, and Other Parameters in Humans. American Journal of Clinical Nutrition, 38, 504-511.

[22] Azadbakht, L., Kimiagar, M., Mehrabi, Y., Esmaillzadeh, A., Hu, F.B. and Willett, W.C. (2007) Soy Consumption, Markers of Inflammation, and Endothelial Function: A Cross-Over Study in Postmenopausal Women with the Metabolic Syndrome. Diabetes Care, 30, 967-973. http://dx.doi.org/10.2337/dc06-2126

[23] Ali, A.A., Velasquez, M.T., Hansen, C.T., Mohamed, A.I. and Bhathena, S.J. (2005) Modulation of Carbohydrate Metabolism and Peptide Hormones by Soybean Isoflavones and Probiotics in Obesity and Diabetes. Journal of Nutritional Biochemistry, 16, 693-699. http://dx.doi.org/10.1016/j.jnutbio.2005.03.011

[24] Azadbakht, L. and Esmaillzadeh, A. (2012) Soy Intake and Metabolic Health: Beyond Isoflavones. Archives of Iranian Medicine, 15, 460-461.

[25] Anderson, J.W., Baird, P., Davis Jr., R.H., Ferreri, S., Knudtson, M., Koraym, A., et al. (2009) Health Benefits of Dietary Fiber. Nutrition Reviews, 67, 188-205. http://dx.doi.org/10.1111/j.1753-4887.2009.00189.x

[26] Meyer, K.A., Kushi, L.H., Jacobs Jr., D.R., Slavin, J., Sellers, T.A. and Folsom, A.R. (2000) Carbohydrates, Dietary Fiber, and Incident Type 2 Diabetes in Older Women. American Journal of Clinical Nutrition, 71, 921-930. 
Scientific Research Publishing (SCIRP) is one of the largest Open Access journal publishers. It is currently publishing more than 200 open access, online, peer-reviewed journals covering a wide range of academic disciplines. SCIRP serves the worldwide academic communities and contributes to the progress and application of science with its publication.

Other selected journals from SCIRP are listed as below. Submit your manuscript to us via either submit@scirp.org or Online Submission Portal.
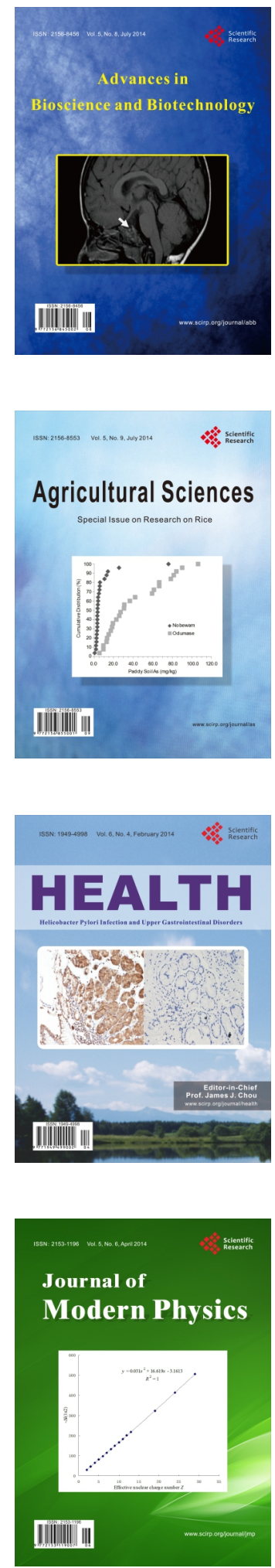
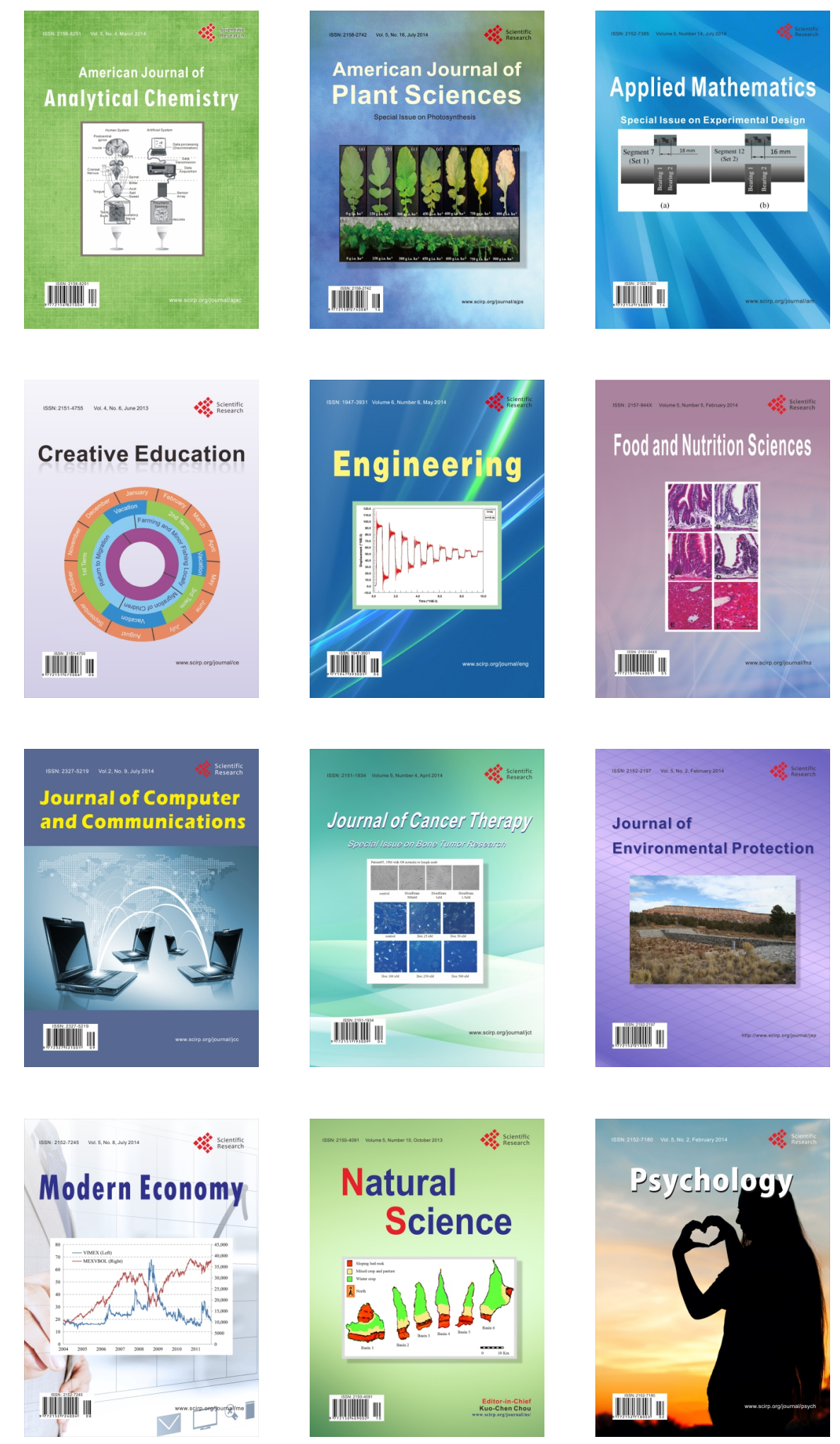